\title{
End-To-End Distribution Function Function of Stiff Polymers for all Persistence Lengths
}

\author{
B. Hamprecht and H. Kleinert \\ Institut für Theoretische Physik, Freie Universität Berlin, \\ Arnimallee 14, D-14195 Berlin, Germany \\ e-mails: bodo.hamprecht@physik.fu-berlin.de e-mails: hagen.kleinert@physik.fu-berlin.de
}

\begin{abstract}
We set up recursion relations for calculating all even moments of the end-to-end distance of a Porod-Kratky wormlike chains in $D$ dimensions. From these moments we derive a simple analytic expression for the end-to-end distribution in three dimensions valid for all peristence lengths. It is in excellent agreement with Monte Carlo data for stiff chains and goes properly over into the Gaussian random-walk distributions for low stiffness.
\end{abstract}

\section{INTRODUCTION}

The main quantity of interest for a stiff polymer in $D$ dimensions is the end-to-end distribution defined by the path integral of a stiff polymer [1]

$\begin{aligned} P_{L}(\mathbf{R}) & \propto \int d^{D} u_{b} \int d^{D} u_{a} \int \mathcal{D}^{D} u \\ & \times \delta^{(D)}\left(\mathbf{R}-\int_{0}^{L} d s \mathbf{u}(s)\right) e^{-(\bar{\kappa} / 2) \int_{0}^{L} d s\left[\mathbf{u}^{\prime}(s)\right]^{2}} .\end{aligned}$

where $\bar{\kappa}$ is the reduced stiffness related to the persistence length $\xi$ by

$$
\bar{\kappa}=\frac{\kappa}{k_{B} T}=(D-1) \frac{\xi}{2},
$$

The unit vectors $\mathbf{u}(s)$ are the tangent vectors of the space curve of the polymer. A Fourier representation of the $\delta$ function brings this to the form

$P_{L}(\mathbf{R}) \propto \int_{-i \infty}^{i \infty} \frac{d^{D} \lambda}{2 \pi i} e^{\bar{\kappa} \boldsymbol{\lambda} \mathbf{R} / 2} \int d^{D} u_{b} \int d^{D} u_{a}\left(\mathbf{u}_{b} L \mid \mathbf{u}_{a} 0\right)^{\boldsymbol{\lambda}}$,

where

$\left(\mathbf{u}_{b} L \mid \mathbf{u}_{a} 0\right)^{\boldsymbol{\lambda}} \equiv \int_{\mathbf{u}(0)=\mathbf{u}_{a}}^{\mathbf{u}(L)=\mathbf{u}_{b}} \mathcal{D}^{D} u e^{-(\bar{\kappa} / 2) \int_{0}^{L} d s\left\{\left[\mathbf{u}^{\prime}(s)\right]^{2}+\boldsymbol{\lambda} \cdot \mathbf{u}(s)\right\}}$

coincides with the euclidean path integral of a point particle of mass $M=\bar{\kappa}$ moving on a unit sphere in an external electric field $\boldsymbol{\lambda}$.

Since $\mathbf{u}(s)$ are unit vectors, the path integral is not solvable exactly, except for zero $\boldsymbol{\lambda}$. It is however, easy to find arbitrarily high even moments of the end-to-end distance of the distribution $P_{L}(\mathbf{R})$ :

$$
\left\langle R^{2 n}\right\rangle \equiv \int d^{D} R R^{2 n} P_{L}(\mathbf{R}) .
$$

The $2 n$th moment of the chain can be obtained directly from the expansion coefficient in powers of $\boldsymbol{\lambda}$ of the integral over (1.4).
In naturla units with $\bar{\kappa}) 1$, the path integral (1.4) solves the Schrödinger equation

$$
\left(-\frac{1}{2} \Delta_{\mathbf{u}}+\frac{1}{2} \boldsymbol{\lambda} \cdot \mathbf{u}+\frac{d}{d \tau}\right)\left(\mathbf{u} \tau \mid \mathbf{u}_{a} 0\right)^{\boldsymbol{\lambda}}=0,
$$

where $\Delta$ is the Laplacian on a unit sphere. The external electric field $\boldsymbol{\lambda}$ may be assumed to point in the $z$ direction, or the $D$ th direction in $D$-dimensions. In the distribution (1.3), only the integrated expression

$$
\psi(z, \tau ; \lambda) \equiv \int d^{D} u_{a}\left(\mathbf{u} \tau \mid \mathbf{u}_{a} 0\right)^{\boldsymbol{\lambda}}
$$

appears, which is a function of $z=\cos \theta$ only, where $\theta$ is the angle between $\mathbf{u}$ and the electric field $\boldsymbol{\lambda}$. Setting $\bar{\kappa}=1$, for a moment, we obtain the simple Schrödinger equation in euclidean time

$$
\hat{H} \psi(z, \tau ; \lambda)=-\frac{d}{d \tau} \psi(z, \tau ; \lambda),
$$

$$
\begin{aligned}
\hat{H} & \equiv \hat{H}_{0}+\lambda \hat{H}_{I}=\frac{1}{2} \Delta+\frac{1}{2} \lambda z \\
& =-\frac{1}{2}\left[\left(1-z^{2}\right) \frac{d^{2}}{d z^{2}}-(D-1) z \frac{d}{d z}\right]+\frac{1}{2} \lambda z
\end{aligned}
$$

Now the desired moments (1.5) can be obtained from the coefficient of $\lambda^{2 n} / 2^{2 n}(2 n)$ ! in the expansion of the integral over (1.7),

$$
f(\tau ; \lambda) \equiv \int_{-1}^{1} d z \psi(z, \tau ; \lambda),
$$

in powers of $\lambda$, evaluated at the euclidean time $\tau=L$.

\section{RECURSIVE SOLUTION OF THE SCHROEDINGER EQUATION.}

The function $f(L ; \lambda)$ has a spectral representation

$$
f(L ; \lambda) \equiv \sum_{l=0}^{\infty} \frac{\int_{-1}^{1} d z \varphi^{(l) \dagger}(z) \exp \left(-E^{(l)} L\right) \varphi^{(l)}(0)}{\int_{-1}^{1} d z \varphi^{(l) \dagger}(z) \varphi^{(l)}(z)}
$$


where $\varphi^{(l)}(z)$ are the solutions of the time-independent Schrödinger equation $\hat{H} \varphi^{(l)}(z)=E^{(l)} \varphi^{(l)}(z)$. Applying perturbation theory to this problem, we start from the eigenstates of the unperturbed Hamiltonian $\hat{H}_{0}=$ $-\Delta / 2$, which are given by the Gegenbauer polynomials $C_{l}^{D / 2-1}(z)$ with the eigenvalues $E_{0}^{(l)}=l(l+D-2) / 2[2]$. Next we set up a recursion scheme for the perturbation expansion of the eigenvalues and eigenfunctions as described in [4]. We begin with a brief review of the method. Starting point is the usual expansion of energy eigenvalues and states in powers of the coupling constant $\lambda$ :

$$
\begin{aligned}
E^{(l)} & =\sum_{j=0}^{\infty} \epsilon_{j}^{(l)} \lambda^{j} \\
\left|\varphi^{(l)}\right\rangle & =\sum_{l^{\prime}, i=0}^{\infty} \gamma_{l^{\prime}, i}^{(l)} \lambda^{i} \alpha_{l^{\prime}}\left|l^{\prime}\right\rangle .
\end{aligned}
$$

The wave functions $\varphi^{(l)}(z)$ are the scalar products $\left\langle z \mid \varphi^{(l)}\right\rangle$. The index $i$ counts the order of the interaction strength $\lambda$. The lowest expansion coefficients of the energy are of course $\epsilon_{0}^{(l)}=E_{0}^{(l)}$. In the second line, we have introduced auxiliary normalization constants $\alpha_{l^{\prime}}$ for convenience to be fixed later. The state vectors $|l\rangle$ of the unperturbed system are normalized to unity, but the state vectors $\left|\varphi^{(l)}\right\rangle$ of the interacting system will be normalized in such a way, that $\left\langle\varphi^{(l)} \mid l\right\rangle=\alpha_{l}$ holds to all orders, implying that

$$
\gamma_{l, i}^{(l)}=\delta_{i, 0} \quad \gamma_{k, 0}^{(l)}=\delta_{l, k}
$$

Inserting the above expansions into the Schrödinger equation, projecting the result onto the base vector $\langle k| \alpha_{k}$, and extracting the coefficient of $\lambda^{i}$, we obtain the relation:

$$
\gamma_{k, i}^{(l)} \epsilon_{0}^{(k)}+\sum_{j=0}^{\infty} \frac{\alpha_{j}}{\alpha_{k}} V_{k, j} \gamma_{j, i-1}^{(l)}=\sum_{j=0}^{i} \epsilon_{j}^{(l)} \gamma_{k, i-j}^{(l)},
$$

where $V_{k, j}=\lambda\langle k|z| j\rangle$ are the matrix elemts of the interaction between unperturbed states. For $i=0$, Eq. (2.5) is satisfied identically. For $i>0$, it leads to the following two recursion relations, one for $k=l$ :

$$
\epsilon_{i}^{(l)}=\sum_{n= \pm 1} \gamma_{l+n, i-1}^{(l)} W_{n}^{(l)}
$$

the other for $k \neq l$ :

$$
\gamma_{k, i}^{(l)}=\frac{\sum_{j=1}^{i-1} \epsilon_{j}^{(l)} \gamma_{k, i-j}^{(l)}-\sum_{n= \pm 1} \gamma_{k+n, i-1}^{(l)} W_{n}^{(l)}}{\epsilon_{0}^{(k)}-\epsilon_{0}^{(l)}},
$$

where only $n=-1$ and $n=1$ contribute to the sums over $n$ since

$$
W_{n}^{(l)} \equiv \frac{\alpha_{l+n}}{\alpha_{l}}\langle l|z| l+n\rangle=0, \text { for } n \neq \pm 1 .
$$

The vanishing of $W_{n}^{(l)}$ for $n \neq \pm 1$ is due to the banddiagonal form of the matrix of the interaction $z$ in the unperturbed basis $|l\rangle$. It is this property which makes the sums in (2.6) and (2.7) finite and leads to recursion relations with a finite number of terms for all $\epsilon_{i}^{(l)}$ and $\gamma_{k, i}^{(l)}$. To calculate $W_{n}^{(l)}$, it is convenient to express $\langle l|z| l+n\rangle$ as matrix elements between unnormalized noninteracting states $\left.\mid l^{\prime}\right\}$ as

$$
\langle l|z| l+n\rangle=\frac{\{l|z| l+n\}}{\sqrt{\{l \mid l\}\{l+n \mid l+n\}}},
$$

where

$$
\{k|F(z)| l\} \equiv \int_{-1}^{1} d z C_{k}^{D / 2-1}(z) F(z) C_{l}^{D / 2-1}(z)\left(1-z^{2}\right)^{(D-3) / 2}
$$

yielding $[5]$

$$
\{l \mid l\}=\frac{2^{4-D} \Gamma(l+D-2) \pi}{l !(2 l+D-2) \Gamma(D / 2-1)^{2}}
$$

Expanding the numerator of (2.9) with the help of the recursion relation for the Gegenbauer polynomials [6]

$$
(l+1) \mid l+1\}=(2 l+D-2) z \mid l\}-(l+D-3) \mid l-1\}
$$

we find the only non-vanishing matrix elements to be

$$
\begin{aligned}
& \{l+1|z| l\}=\frac{l+1}{2 l+D-2}\{l+1 \mid l+1\}, \\
& \{l-1|z| l\}=\frac{l+D-3}{2 l+D-2}\{l-1 \mid l-1\} .
\end{aligned}
$$

Inserting these together with (2.11) into (2.9) gives

$$
\langle l|z| l-1\rangle=\sqrt{\frac{l(l+D-3)}{(2 l+D-2)(2 l+D-4)}},
$$

and a corresponding result for $\langle l|z| l+1\rangle$. We now fix the normalization constants $\alpha_{l^{\prime}}$ by setting

$$
W_{1}^{(l)}=\frac{\alpha_{l+1}}{\alpha_{l}}\langle l|z| l+1\rangle=1
$$

for all $l$, which determines the ratios

$$
\frac{\alpha_{l}}{\alpha_{l+1}}=\langle l|z| l+1\rangle=\sqrt{\frac{(l+1)(l+D-2)}{(2 l+D)(2 l+D-2)}} .
$$

Setting further $\alpha_{1}=1$, we obtain

$$
\alpha_{l}=\left[\prod_{j=1}^{l} \frac{(2 l+D-2)(2 l+D-4)}{l(l+D-3)}\right]^{1 / 2}
$$


Using this we find from (2.8) the remaining nonzero $W_{n}^{(l)}$ for $n=-1$ :

$$
W_{-1}^{(l)}=\frac{l(l+D-3)}{(2 l+D-2)(2 l+D-4)} .
$$

We are now ready to solve the recursion relations (2.6) and (2.7) for $\gamma_{k, i}^{(l)}$ and $\epsilon_{i}^{(l)}$ order by order in $i$. For the initial order $i=0$, the values of the $\gamma_{k, i}^{(l)}$ are given by Eq. (2.4). The coefficients $\epsilon_{i}^{(l)}$ are equal to the unperturbed energies $\epsilon_{0}^{(l)}=E_{0}^{(l)}=l(l+D-2) / 2$. For each $i=1,2,3, \ldots$, there is only a finite number of non-vanishing $\gamma_{k, j}^{(l)}$ and $\epsilon_{j}^{(l)}$ with $j<i$ on the right-hand sides of (2.6) and (2.7) which allows us to calculate $\gamma_{k, i}^{(l)}$ and $\epsilon_{i}^{(l)}$ on the left-hand sides. In this way it is easy to find the perturbation expansions for the energy and the wave functions to high orders.

Inserting the resulting expansions (2.2) and (2.3) into Eq. (2.1), only the totally symmetric parts in $\varphi^{(l)}(z)$ will survive the integration in the numerators, i.e., we may insert only

$$
\varphi_{\mathrm{symm}}^{(l)}(z)=\left\langle z \mid \varphi_{\mathrm{symm}}^{(l)}\right\rangle=\sum_{i=0}^{\infty} \gamma_{0, i}^{(l)} \lambda^{i}\langle z \mid 0\rangle .
$$

The denominators of 2.1 become explicitly $\sum_{l^{\prime}, i}\left|\gamma_{l^{\prime}, i}^{(l)} \alpha_{l^{\prime}}\right|^{2} \lambda^{2 i}$, where the summation over $i$ is limited by power of $\lambda^{2}$ up to which we want to carry the perturbation series; also $l^{\prime}$ is restricted to a finite number of terms only, because of the band-diagonal structure of the $\gamma_{l^{\prime}, i}^{(l)}$.

Extracting the coefficients of the power expansion in $\lambda$ from (2.1) we obtain all desired moments of the end-toend distribution, the lowest two being, after reinserting $\bar{\kappa}$ from (1.2),

$$
\begin{gathered}
\left\langle R^{2}\right\rangle=2\left\{\xi L-\xi^{2}\left[1-e^{-L / \xi}\right]\right\} . \\
\left\langle R^{4}\right\rangle=\frac{4(D+2)}{D} L^{2} \xi^{2}-8 L \xi^{3}\left(\frac{D^{2}+6 D-1}{D^{2}}-\frac{D-7}{D+1} e^{-L / \xi}\right) \\
+4 \xi^{4}\left[\frac{D^{3}+23 D^{2}-7 D+1}{D^{3}}-2 \frac{(D+5)^{2}}{(D+1)^{2}} e^{-L / \xi}\right. \\
\left.+2 \frac{(D-5)^{5}}{D^{3}(D+1)^{2}} e^{-2 D L /(D-1) \xi}\right] .
\end{gathered}
$$

The calculation of higher moments is straightforward with a Mathematica program, which we have made available on the internet in notebook form [7].

\section{FROM MOMENTS TO END-TO-END DISTRIBUTION IN $D=3$ DIMENSIONS.}

The moments can now be used to recover the experimentally accessible end-to-end distribution of the polymers for various degrees of stiffness. We parameterize the distribution with an analytic form

$$
P_{L}(\mathbf{R}) \propto r^{k+2}\left(1-r^{\beta}\right)^{m}, \quad r \equiv R / L .
$$

whose moments are

$$
\left\langle R^{2 n}\right\rangle=\frac{\Gamma\left(\frac{3+k+2 n}{\beta}\right) \Gamma\left(\frac{3+k}{\beta}+m+1\right)}{\Gamma\left(\frac{3+k}{\beta}\right) \Gamma\left(\frac{3+k+2 n}{\beta}+m+1\right)}
$$

We now adjust the three parameters $k, \beta$, and $m$ to fit the three most important moments of the distribution exactly, ignoring all others. If the distances were distributed uniformly over the interval $r \in[0,1]$, the moments would be $\left\langle R^{2 n}\right\rangle^{\text {flat }}=1 /(2 n+2)$. Comparing our exact moments $\left\langle R^{2 n}\right\rangle(\xi)$ with the flat ones, we find that $\left\langle R^{2 n}\right\rangle(\xi) /\left\langle R^{2 n}\right\rangle^{\text {flat }}$ has a maximum for $n$ close to $n_{\max }(\xi) \equiv 4 \xi$. The most important moments turn out to be the ones with $n=n_{\max }(\xi)$ and $n=n_{\max }(\xi) \pm 1$. If $n_{\max }(\xi) \leq 1$ we use the lowest even moments $\left\langle R^{2}\right\rangle,\left\langle R^{4}\right\rangle$, and $\left\langle R^{6}\right\rangle$. With these adjustments the resulting distributions are shown in Fig. 1 for various persistence lengths $\xi$. They are in excellent agreement with the Monte Carlo data (symbols) obtained by Wilhelm and Frey [8], and better than their one-loop perturbative results (thin curves) which are good only for very stiff polymers. For the small persistence lengths $\xi=1 / 400,1 / 100,1 / 30$, the curves are well approximated by Gaussian random chain distributions on a lattice with lattice constant $a_{\text {eff }}=2 \xi$ which ensures that $a_{\text {eff }}=2 \xi$ the lowest moments $\left\langle R^{2}\right\rangle=a_{\mathrm{eff}} L$ are properly fitted.

Acknowledgment

This work was partially supported by ESF COSLAB Program and by the Deutsche Forschungsgemeinschaft under Grant Kl-256. 


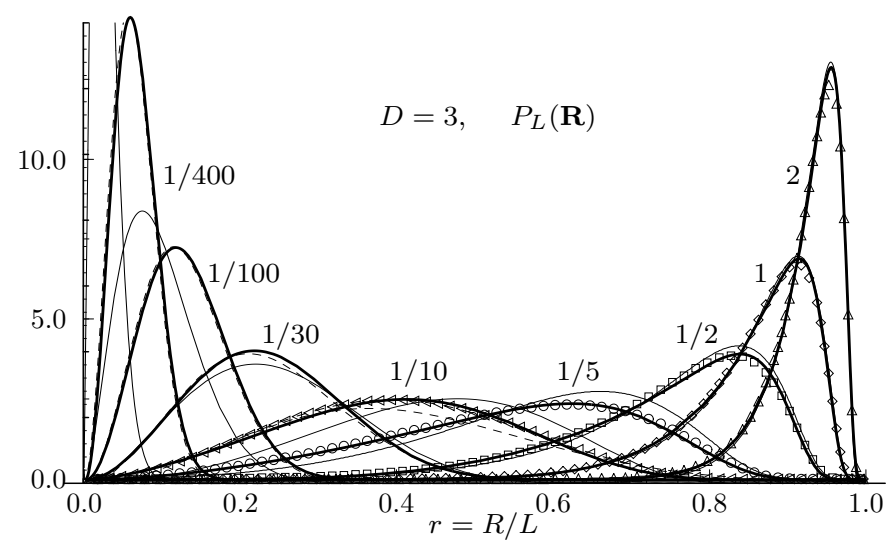

FIG. 1: Distribution of the end-to-end distances of polymer for different stiffnesses, parametrized by the persistence lengths $\xi=1 / 400,1 / 100,1 / 30,1 / 10,1 / 5,1 / 2,1,2$. They are compared with the Monte Carlo calculations of Wilhelm and Frey 8] (symbols) and with his large-stiffness one-loop perturbative results (thin curves). For the small stiffnesses $\xi=1 / 400,1 / 100,1 / 30$, the curves are well approximated by Gaussian random chain distributions on a lattice with lattice constant $a_{\mathrm{eff}}=2 \xi$ which ensures that $a_{\mathrm{eff}}=2 \xi$ the lowest moments $\left\langle R^{2}\right\rangle=a_{\text {eff }} L$ are properly fitted (dashed curves).

[1] H.Kleinert, Path Integrals in Quantum Mechanics, Statistics and Polymer Physics, World Scientific, Singapore, 1995. (http://www. physik.fu-berlin. $\mathrm{de} /{ }^{\sim} \mathrm{kleinert} / \mathrm{b} 5$ ).

[2] See Subsection 15.7.2 in the textbook [1].

[3] H.Kleinert, V.Schulte-Frohlinde Critical Properties of $\Phi^{4}$-Theories, World Scientific, Singapore, 2001 (http://www.physik.fu-berlin.de/ ${ }^{\sim} k l e i n e r t / b 8$ ).

[4] See the Appendices of Chapter 3 in the textbook [1], and the article by B. Hamprecht and A. Pelster Fluctuating Paths and Fields - Festschrift Dedicated to Hagen Klein- ert on the Occasion of his 60th Birthday, Eds. W. Janke, A. Pelster, H.-J. Schmidt, and M. Bachmann, World Scientific, Singapore, 2001, p. 347.

[5] I.S. Gradshteyn and I.M. Ryzhik, Table of Integrals, Series, and Products, Academic Press, New York, 1980, Formulas 7.313.

[6] ibid., Formula 8.933.1.

[7] The "Mathematica" notebook can be obtained form from http://www.physik.fu-berlin.de/ ₹kleinert/b5/pgm15

[8] J. Wilhelm and E. Frey, Phys. Rev. Lett. 77, 2581 (1996). 